(c) 2017 IEEE. Personal use of this material is permitted. Permission from IEEE must be obtained for all other users, including reprinting/ republishing this material for advertising or promotional purposes, creating new collective works for resale or redistribution to servers or lists, or reuse of any copyrighted components of this work in other works. 


\title{
Variable Structure Control for Three-Phase LCL-Filtered Inverters using a Reduced Converter Model
}

\author{
Ramon Guzman, Luis Garcia de Vicuña, Miguel Castilla, Jaume Miret,Member, IEEE, Jordi de la Hoz
}

\begin{abstract}
This paper presents a new concept in active damping techniques using a reduced model of a LCLfiltered grid connected inverter. The presence of the LCL filter complicates the design of the inverter control scheme, particularly when uncertainties in the system parameters, especially in the grid inductance, are considered. The proposed control algorithm is addressed to overcome such difficulties using a reduced model of the inverter in a state observer. In this proposal, two of the three state variables of the system are obviated from the physical inverter model and only the inverter side current is considered. Therefore, the inverter side current can be estimated emulating the case of an inverter with only one inductor, thus eliminating the resonance problem produced by the LCL filter. Besides, in the case of a distorted grid, the method allows to estimate the voltages at the point of common coupling free of noise and distortion without using any PLL-based synchronization algorithm. This proposal provides the following features to the closed-loop system: 1) robust and simple active damping control under system parameters deviation, 2) robustness against grid voltage unbalance and distortion, and 3) an important reduction in the computational load of the control algorithm which allows to increase the switching frequency. To complete the control scheme, a theoretical stability analysis is developed considering the effect of the observer, the system discretization and the system parameters deviation. Experimental and comparative evaluation results are presented to validate the effectiveness of the proposed control scheme.
\end{abstract}

Index Terms-Sliding mode control (SMC), LCL filter, Kalman filter (KF), Reduced model

\section{INTRODUCTION}

$\mathbf{N}$ OWADAYS the energy sector is moving to the sector of smart grids (SGs) with aim of maximizing the penetration of distributed generation (DG) [1], [2]. The main objective is to improve the overall grid stability, reliability and efficiency. For this reason, the design of robust controllers for DG units is the main concern of the researchers. Usually, most of these DG units are interfaced to the utility grid by means of a voltage source inverter (VSI) [3] which main function is to extract the maximum power from the energy source and inject it to the utility grid as active power. Some amount of reactive power can also be injected according to some specific requirements [4]. Conventionally, the VSIs are connected to the utility grid through a LCL filter since this option yields to a better attenuation of the switching harmonics. However, this approach leads to an inherent resonance problem which can be overcome using an active or passive damping solution.
Several damping solutions have been established to damp the resonance of the LCL filter. In fact, there are two different techniques. The passive damping methods are based on introducing additional passive elements on the filter. Usually resistors are employed, causing power losses and reducing the overall system efficiency [5], [6]. Here, it is important to remark that these losses may be unacceptable in some applications, for instance in wind turbines, since they operate typically at $30 \%$ of the converter nominal power [7]. Alternatively, active damping can be applied. The active damping methods are aimed to improve the system efficiency and they are based on modifying the closed loop dynamics by adding a digital filter in the control loop. This filter is generally a notch filter which is tuned according to the value of the LCL filter resonance frequency [8].

In some cases, the distance between DG units is unknown, and consequently a large set of grid impedance should be considered [6]. Under this condition, the uncertainty of the grid impedance at the point of common coupling (PCC), and also deviations in the system parameters, are important concerns to be addressed. These issues directly affect the resonance frequency value of the LCL filter and might lead the current controller to an unstable dynamics due to a detuning of the digital filter.

Then, auto-tuning procedures can be adopted in order to solve this problem, as for instance the solution proposed in [9]. However, in order to avoid these self-tuning procedures, different solutions can be found in the literature using feedback controllers [10], [11]. These controllers are usually based on a state observer where an accurate power converter model is used to estimate the state variables. An interesting solution with a high robustness against grid inductance variations is presented in [12]. Here, a virtual resistor is introduced in the converter model in order to emulate a damping resistor.The main advantage of this work is the use of sliding mode control (SMC), which provides a high robustness against system parameters deviations and external disturbances. Besides, a fast dynamic response is achieved [13]. However, the main drawback of this method is the high computational load which limits the value of maximum switching frequency.

Unlike these control methods, the sliding-mode observerbased control presented in this paper uses a reduced statespace model which does not rely on the grid inductance value. Besides, the proposed solution does not need any self-tuning procedure or adaptive techniques to provide robustness against 


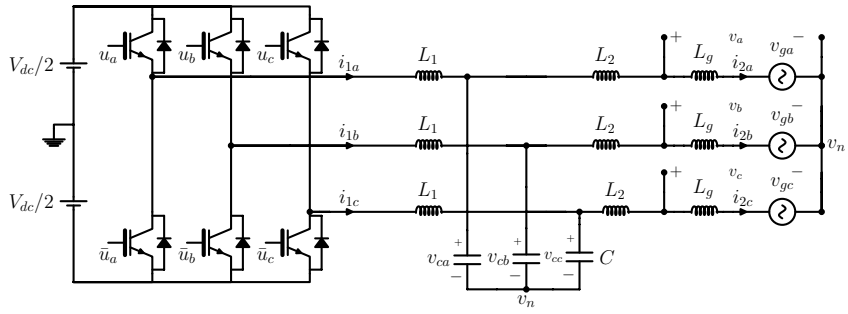

Fig. 1. Circuit diagram of three-phase grid-connedcted inverter with LCL-filter

parameters deviations, even in the case of severe changes in the grid impedance. Compared with [12] or other complex strategies based on SMC, the computational load of the control algorithm is clearly reduced. This fact allows to increase the switching frequency in the SMC operation.

In order to analyze and design the proposed robust controller the following steps will be performed:

1) A reduced state-space model of the VSI is proposed to design three independent current controllers in natural reference frame. In this model only the inverter side current is considered.

2) Three simple switching surfaces will be proposed using estimated variables obtained from a state observer, in this case, a Kalman filter (KF).

3) A complete stability analysis will be performed taking into account the effect of the KF, the system discretization and the deviation in the system parameters. This analysis will prove the system robustness.

4) Finally, experimental results will be reported to prove the aforementioned properties, including the controller response against a distorted grid and voltage sags.

The properties of the proposed control method are: 1) high robustness against system parameters deviation, specially for grid inductance changes, 2) a tuningless control method which ensures robustness when the system parameters or the grid impedance vary, and 3) a fast dynamic response against sudden changes in the current references.

As a comparison with other previous SMC methods [12], [14], [15], the use of a reduced model allows to stabilize the system. The main advantage is that the computational time used by the algorithm is clearly reduced and as a consequence the maximum switching frequency can be increased. Besides, this technique is not only useful in this approach, but also can be applied to other power converters in order to reduce the complexity of the control algorithm.

The paper is organized as follows. In Section II a bilinear model of the VSI with LCL filter is presented. Section III presents the proposed control system. A stability analysis is presented in section IV. Simulation and experimental results are presented in section V. A comparative analysis regarding the computational time is presented in section VI. Finally, section VII concludes the paper.

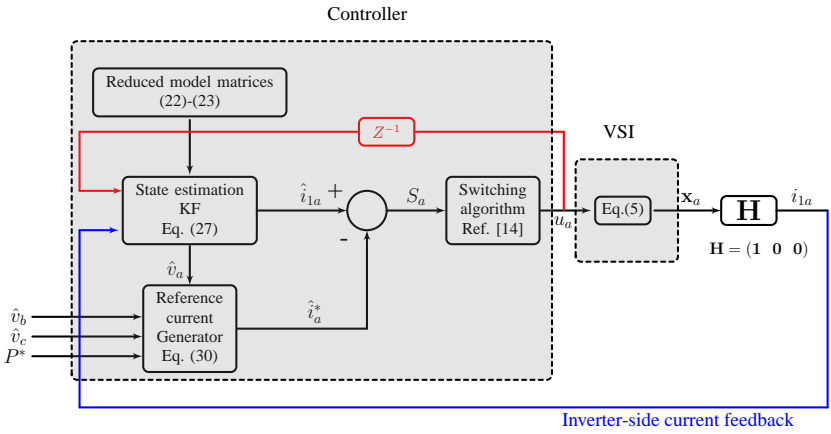

Fig. 2. Proposed control system for phase-leg a

\section{Physical Model equations for a threE-PHASE GRID-CONNECTED VSI WITH LCL FILTER}

Fig.1 shows a three-phase grid-connected VSI with LCL filter, where the grid impedance is considered pure inductive. The VSI equations can be obtained from the circuit as follows:

$$
\begin{aligned}
L_{1} \frac{d \mathbf{i}_{1}}{d t} & =\frac{V_{d c}}{2} \mathbf{u}-\mathbf{v}_{c}-v_{n} \mathbf{1} \\
C \frac{d \mathbf{v}_{c}}{d t} & =\mathbf{i}_{1}-\mathbf{i}_{2} \\
\left(L_{2}+L_{g}\right) \frac{d \mathbf{i}_{2}}{d t} & =\mathbf{v}_{c}-\mathbf{v}_{g} .
\end{aligned}
$$

where $\mathbf{i}_{1}=\left[\begin{array}{lll}i_{1 a} & i_{1 b} & i_{1 c}\end{array}\right]^{T}$ is the inverter-side current vector, $\mathbf{i}_{2}=\left[\begin{array}{lll}i_{2 a} & i_{2 b} & i_{2 c}\end{array}\right]^{T}$ is the grid-side current vector, $\mathbf{v}_{c}=\left[\begin{array}{lll}v_{c a} & v_{c b} & v_{c c}\end{array}\right]^{T}$ is the capacitor voltage vector, $\mathbf{v}_{g}=$ $\left[\begin{array}{lll}v_{g a} & v_{g b} & v_{g c}\end{array}\right]^{T}$ is the grid voltage vector, $\mathbf{u}=\left[\begin{array}{lll}u_{a} & u_{b} & u_{c}\end{array}\right]^{T}$ is the control signals vector, $v_{n}$ is the voltage at the neutral point which is expressed as:

$$
v_{n}=\frac{V_{d c}}{6}\left(u_{a}+u_{b}+u_{c}\right)
$$

and 1 is a column vector defined as $\left[\begin{array}{lll}1 & 1 & 1\end{array}\right]^{T}$.

The above differential equations can be rewritten as a discrete state-space model where the process and measurement discrete equations for each phase-leg $i$, where $i \in\{a, b, c\}$, are expressed as follows:

$$
\begin{gathered}
\mathbf{x}_{i}(k+1)=\mathbf{A} \mathbf{x}_{i}(k)+\mathbf{B} u_{i}(k)+\mathbf{D} v_{g i}(k)+\mathbf{E} v_{n}(k) \\
y_{i}(k)=\mathbf{H} \mathbf{x}_{i}(k)
\end{gathered}
$$

being $\mathbf{x}_{i}(k)=\left(\begin{array}{lll}i_{1 i} & v_{c i} & i_{2 i}\end{array}\right)$ the space-state vector, $\mathbf{H}$ the output matrix defined as

$$
\mathbf{H}=\left(\begin{array}{lll}
1 & 0 & 0
\end{array}\right)
$$

and $\mathbf{A}, \mathbf{B}, \mathbf{D}$ and $\mathbf{E}$ the system discrete matrices which are computed using the first order approximation as follows:

$$
\begin{gathered}
\mathbf{A} \cong \mathbf{I}+\mathbf{A}_{c} T_{s}=\left(\begin{array}{ccc}
1 & -T_{s} / L_{1} & 0 \\
T_{s} / C & 1 & -T_{s} / C \\
0 & T_{s} /\left(L_{2}+L_{g}\right) & 1
\end{array}\right) \\
\mathbf{B} \cong \mathbf{B}_{c} T_{s}=\left(\begin{array}{lll}
V_{d c} T_{s} / 2 L_{1} & 0 & 0
\end{array}\right)^{T} \\
\mathbf{D} \cong \mathbf{D}_{c} T_{s}=\left(\begin{array}{ccc}
0 & 0 & -T_{s} /\left(L_{2}+L_{g}\right)
\end{array}\right)^{T} \\
\mathbf{E} \cong \mathbf{E}_{c} T_{s}=\left(\begin{array}{lll}
-T_{s} / L_{1} & 0 & 0
\end{array}\right)^{T}
\end{gathered}
$$



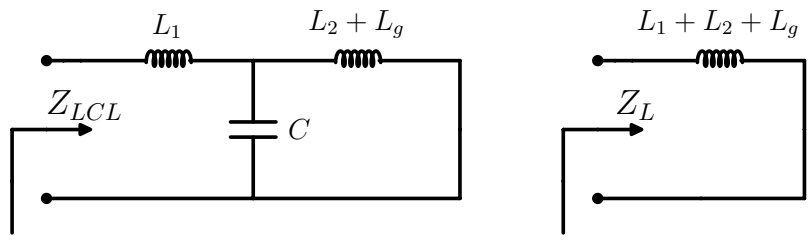

Fig. 3. Input impedances of the LCL filter, $Z_{L C L}$ and for the $\mathrm{L}$ filter model, $Z_{L}$

with $T_{s}$ the sampling time and $\mathbf{A}_{c}, \mathbf{B}_{c}, \mathbf{D}_{c}$ and $\mathbf{E}_{c}$ the continuous system matrices.

The above system discrete equations will be used in the stability analysis section in order to validate our control proposal.

\section{Proposed Control System}

The proposed control scheme is oriented to achieve a robust active damping algorithm using a sliding-mode control with a reduced model. Fig.2 depicts the proposed control scheme for phase-leg $a$, which has the same structure for the phases $b$ and $c$. The proposed controller is based on a state observer, a $\mathrm{KF}$, which uses a reduced VSI model in order to estimate the inverter-side current, the PCC voltage and its quadrature. As shown in the figure, the reference current is obtained according to the desired active power, and also using the estimated PCC voltages.

Once the reference is computed, the reference current and the estimated inverter-side current are used in a sliding surface, and a switching algorithm obtains the control signal $u_{a}$. This algorithm is presented in [14], [15] and leads to obtain a quasi-fixed switching frequency. Finally, the generated control signal, is applied to control the phase-leg $a$ of the VSI, generating a current without oscillations. In order to validate this control proposal, a complete stability analysis will be performed including the KF effect, which uses the reduced space-state model presented in the next section.

\section{A. Reduced state-space model of the converter}

Fig. 3 shows the filter equivalent circuits of a $\mathrm{L}$ and LCL structures from the point of view of its input impedance. By appling the Laplace transform to equations (1)-(3), the input impedance of the LCL filter presented in Fig.3 can be obtained as follows:

$$
Z_{L C L}=\frac{L_{1} C\left(L_{2}+L_{g}\right) s^{3}+\left(L_{1}+L_{2}+L_{g}\right) s}{C\left(L_{2}+L_{g}\right) s^{2}+1}
$$

while the input impedance for the L filter topology in Fig. 3 is expressed as:

$$
Z_{L}=\left(L_{1}+L_{2}+L_{g}\right) s
$$

It can be easily found that $Z_{L C L} \cong Z_{L}$ when $\omega_{o}<<\omega_{\text {res }}$, being $\omega_{o}$ the grid frequency. Note that when a SMC is used, the resonance frequency can be obtained according to [12]:

$$
\omega_{\text {res }}=1 / \sqrt{\left(L_{2}+L_{g}\right) C} .
$$

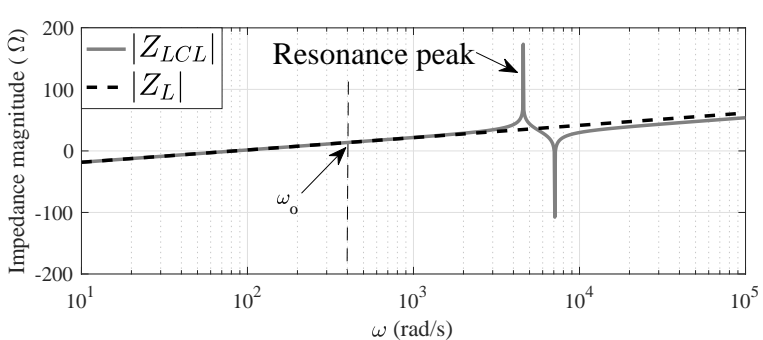

(a)

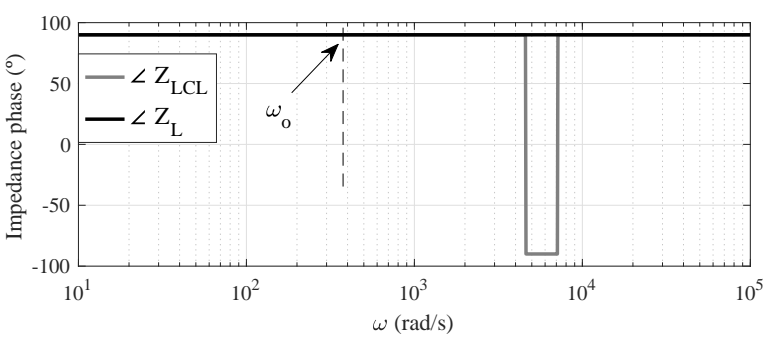

(b)

Fig. 4. Bode plot of the input impedance in the case of the physical model and in the case of the reduced model: a) magnitude, and b) phase.

TABLE I

SYSTEM PARAMETERS

\begin{tabular}{lcc}
\hline \hline Description & Symbol & Value \\
\hline Grid voltage & $V_{g r i d}$ & $110 \mathrm{~V}$ \\
Nominal dc-link voltage & $V_{d c o}$ & $450 \mathrm{~V}$ \\
Nominal filter input inductance & $L_{1 o}$ & $5 \mathrm{mH}$ \\
Nominal filter capacitor & $C_{o}$ & $6.8 \mathrm{uF}$ \\
Nominal filter output inductance & $L_{2 o}$ & $2 \mathrm{mH}$ \\
Grid inductance & $L_{g}$ & $0.5 \mathrm{mH}$ \\
Sampling frequency & $f_{s}$ & $60 \mathrm{kHz}$ \\
Grid frequency & $f_{o}$ & $60 \mathrm{~Hz}$ \\
Switching frequency & $f_{s w}$ & $6 \mathrm{kHz}$ \\
Active power & $P^{*}$ & $1.5 \mathrm{~kW}$ \\
Reactive power & $Q^{*}$ & $0 \mathrm{kVAr}$ \\
Single phase system noise power & $R_{i}(k)$ & $0.26 V^{2}$ \\
\hline
\end{tabular}

According the the aforementioned approximations, the effect of the capacitor can be neglected in the input impedance computation which leads to accomplish that $\mathbf{i}_{1} \cong \mathbf{i}_{2}$. For a better understanding, an impedance Bode plot is represented in Fig.4 using the system parameters listed in Table I. It can be seen that the behavior of both impedances are similar at the frequency $\omega_{o}$.

Using the aforementioned approximations, and from equation (3), the capacitor voltage vector can be rewritten as follows:

$$
\mathbf{v}_{c}=\left(L_{1}+L_{g}\right) \frac{d \mathbf{i}_{1}}{d t}+\mathbf{v}_{g}
$$

Now, by replacing (15) in (1), the following differential equation can be found:

$$
\left(L_{1}+L_{2}+L_{g}\right) \frac{d \mathbf{i}_{1}}{d t}=\frac{V_{d c}}{2} \mathbf{u}-\mathbf{v}_{g}-v_{n} \mathbf{1}
$$

Equation (16) describes the current dynamics of a VSI with only one inductor. According to the Bode plots showed before, 


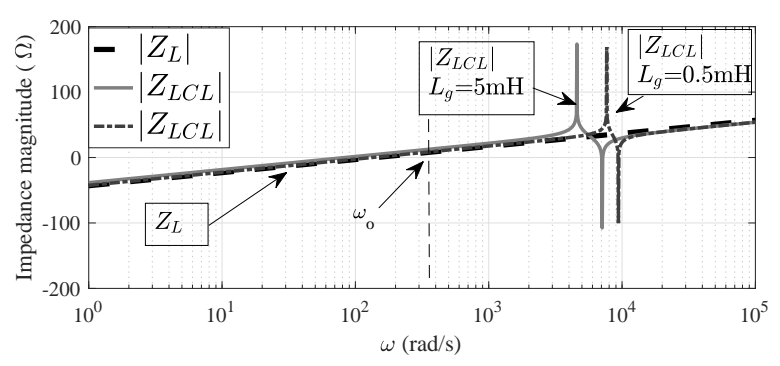

Fig. 5. Bode plot of the input impedance magnitude in the case of the physical model for two different values of the grid inductance, and in the case of the proposed reduced model.

the impedance of both filters is similar at the grid frequency. Therefore, at the frequency of $\omega_{o}$ the dynamics of $\mathbf{i}_{1}$ is approximated to the dynamics of $\mathbf{i}_{2}$. This idea will be applied to derive the proposed reduced model according to the following premises:

1) Since the sliding-mode controller is designed in natural frame, the three current controllers are cross-coupled through the neutral point voltage (see eq.(4)). For this reason, and in order to eliminate these interferences between controllers, the neutral point voltage expression is not considered in the reduced model [12].

2) In order to achieve active damping dynamics, the model only consideres the inverter-side current state variable. The capacitor voltage and the grid-side current are obviated in this model. With this consideration the inverterside current is estimated as in the case of a VSI with only one inductor where the resonance does not exist.

3) The voltage at the PCC and its quadrature are estimated, instead of using the measured grid voltage. This fact leads to a version of the PCC voltage free of noise and distortion even in the case of a distorted grid. Besides, the proposed model is independent of the grid inductance [14], [16].

According to the aforementioned conditions, the proposed reduced model can be represented as follows:

$$
\begin{aligned}
\left(L_{1 o}+L_{2 o}\right) \frac{d \mathbf{i}_{1}}{d t} & =\frac{V_{d c o}}{2} \mathbf{u}-\mathbf{v} \\
\frac{d \mathbf{v}}{d t} & =\omega_{o} \mathbf{v}^{\perp} \\
\frac{d \mathbf{v}^{\perp}}{d t} & =-\omega_{o} \mathbf{v}
\end{aligned}
$$

where $L_{1 o}$ and $L_{2 o}$ are the inductors nominal values, $\omega_{o}$ the grid angular frequency, $V_{d c o}$ the nominal dc-link voltage, and $\mathbf{v}$ and $\mathbf{v}^{\perp}$ the voltage at the PCC and its quadrature respectively.

In order to validate the reduced model, Fig.5 shows the input impedance magnitude of the physical LCL filter for two different values of $L_{g}, 0.5 \mathrm{mH}$ and $5 \mathrm{mH}$ respectively. The input impedance magnitude in the case of the reduced model regarding to the nominal values $L_{1 o}$ and $L_{2 o}$ is also depicted. The Figure shows that the impedance magnitude of proposed model matches with good accuracy with the magnitude impedance of the physical model for both values of the grid impedance. Note that for the proposed reduced model it is not necessary to know the value of the grid inductance, which makes the system robust against changes in this parameter.

\section{B. Proposed Model discretization and Kalman filter adap- tive equation}

For the digital implementation of the control algorithm, this model is discretized in order to be used in a KF algorithm. The reduced discrete space-state model, where the symbol ^ denotes estimated variables, is represented as follows:

$$
\begin{aligned}
\hat{\mathbf{x}}_{i}(k+1) & =\hat{\mathbf{A}} \hat{\mathbf{x}}_{i}(k)+\hat{\mathbf{B}} u_{i}(k)+\boldsymbol{\eta}_{i}(k) \\
y_{i}(k) & =\mathbf{H} x_{i}(k)+\mathbf{w}_{i}(k)
\end{aligned}
$$

being

$$
\begin{gathered}
\hat{\mathbf{A}}=\left(\begin{array}{ccc}
1 & -T_{s} /\left(L_{1 o}+L_{2 o}\right) & 0 \\
0 & 1 & T_{s} \omega_{o} \\
0 & -T_{s} \omega_{o} & 1
\end{array}\right) \\
\hat{\mathbf{B}}=\left(\begin{array}{ccc}
V_{d c o} T_{s} / 2\left(L_{1 o}+L_{2 o}\right) & 0 & 0
\end{array}\right)^{T} \\
\hat{\mathbf{x}}_{i}(k)=\left(\begin{array}{lll}
\hat{i}_{1 i} & \hat{v}_{i} & \hat{v}_{i}^{\perp}
\end{array}\right)^{T}
\end{gathered}
$$

and $\boldsymbol{\eta}_{i}(k)$ and $\mathbf{w}_{i}(k)$ the process and the measurement noise vectors respectively, which defines the following noise and process covariance matrices:

$$
\begin{aligned}
& \mathbf{R}_{i}(k)=E\left\{\mathbf{w}_{i}(k) \mathbf{w}_{i}^{T}(k)\right\} \\
& \mathbf{Q}_{i}(k)=E\left\{\boldsymbol{\eta}_{i}(k) \boldsymbol{\eta}_{i}^{T}(k)\right\}
\end{aligned}
$$

To design the observer-based sliding-mode control a KF is proposed. According to the KF features, a KF-based observer is optimum in presence of noise [14] and improves the dynamics of the closed-loop system in noisy environments. For this reason, the proposed method is focused on the statespace model defined by (20)-(21) used in a KF algorithm. This model forces the estimated inverter-side currents to get similar dynamics as in the case of a VSI with only one inductor. This fact leads to a damped dynamics of the estimated currents. Finally, the sliding-mode control uses these estimated currents in three decoupled sliding mode controllers which allows to fix the switching frequency [14].The main properties and the implementation of the KF are well explained in [17] so only the adaptive equation used for the estimation is given in this section.

The equation for the estate estimation is expressed as follows:

$$
\hat{\mathbf{x}}_{i}(k+1)=\hat{\mathbf{A}} \hat{\mathbf{x}}_{i}(k)+\hat{\mathbf{B}} u_{i}(k)+\mathbf{L}_{i}(k)\left(i_{1 i}(k)-\hat{i}_{1 i}(k)\right)
$$

where the measured inverter side current is used to compute the estimation error, and the Kalman gain is computed using the following expression:

$$
\mathbf{L}_{i}(k)=\mathbf{P}_{i}(k) \mathbf{H}^{T}\left(\mathbf{H P}_{i}(k) \mathbf{H}^{T}+\mathbf{R}_{i}(k)\right)^{-1}
$$

where $\mathbf{P}_{i}$ the error covariance matrix. Note that Kalman gain is computed in order to minimize the noise in the estimation which algorithm is explained in [14], [17]. 


\section{Sliding surfaces with active damping capability}

As it was explained, in order to achieve a damped dynamics the estimated inverter-side current can be used in a slidingmode controller. Then, the following sliding surface for each phase-leg $i$ is proposed:

$$
S_{i}=\hat{i}_{1 i}-\hat{i}_{i}^{*}
$$

where $\hat{i}_{i}^{*}$ is the reference current, which can be computed according to the active and reactive powers references:

$$
\hat{i}_{i}^{*}=\frac{P^{*}}{|\hat{\mathbf{v}}|^{2}} \hat{v}_{i}+\frac{Q^{*}}{\sqrt{3}|\hat{\mathbf{v}}|^{2}} \hat{v}_{i}^{\perp} .
$$

From (29) and (30), the expression of the sliding surface can be rewritten as follows:

$$
S_{i}=\mathbf{C}^{T} \hat{\mathbf{x}}_{i}
$$

where

$$
\mathbf{C}^{T}=\left(\begin{array}{l}
1-\frac{P^{*}}{|\hat{\mathbf{v}}|^{2}}-\frac{Q^{*}}{\sqrt{3}|\hat{\mathbf{v}}|^{2}}
\end{array}\right)
$$

It must be noticed the use of the estimated PCC voltages in the the reference currents computation. This fact allows to reduce the current distortion even in the case of a highly distorted grid [15], [16].

On the other hand, the existence condition of a sliding mode controller is given by the following inequality:

$$
\dot{S}_{i} S_{i}<0
$$

Now, defining the control variable $u_{i}$ as

$$
u_{i}=\left\{\begin{array}{lll}
u_{i}^{+} & \text {if } & S_{i}>0 \\
u_{i}^{-} & \text {if } & S_{i}<0
\end{array}\right.
$$

where $u_{i} \in\{1,-1\}$, and taking into account (17), (29) and (33) we can obtain :

$$
\frac{v_{d c}}{2 L_{1}}\left(u_{i}^{-}-u_{i}^{+}\right)<0
$$

which allows us to determine the switching action:

$$
u_{i}=\left\{\begin{array}{lll}
u_{i}^{+}=1 & \text { if } & S_{i}>0 \\
u_{i}^{-}=-1 & \text { if } & S_{i}<0
\end{array}\right.
$$

\section{STABILITY ANALYSIS}

To conclude with the robust controller design, a stability analysis is performed in this section in order to validate the proposed control method. The stability of the system will be also analyzed accounting for the system parameters deviation. The system stability is ensured if the eigenvalues of the closed loop system are inside the unity circle in the $z$-plane.

The three-phase system has nine poles, each one related to one state variable. Due to the decoupling introduced by the KF with the proposed model, a decoupling between controllers is achieved and the three-phase system can be considered as three independent single-phase systems from the control viewpoint [14]. Therefore, only the stability of a single-phase system is analyzed, and as a consequence, only three poles due to the LCL filter are considered. However, the state observer will add three additional poles. Then, the system together with the observer will have a total of six poles (see Fig.6(b)).
While the system is in sliding regime, it is found that there is one pole fixed at the origin $(z=0)$ [18]. The position of the remaining poles will depend on the model parameters and the Kalman filter gain. The next subsections are dedicated to find the closed-loop equations.

\section{A. Discrete time equivalent control deduction}

The first step is to find the so-called "equivalent control", $\hat{u}_{i e q}$. The equivalent control expression will be used in the next subsection in order to obtain the closed-loop system equations to analyze the system stability.

Then, assuming that the system is in sliding regime, the control signal $u_{i}$ can be substituted by $\hat{u}_{i e q}$ [19]. With this assumption the reduced state-space model for the estimated variables can be written as follows:

$\hat{\mathbf{x}}_{i}(k+1)=\hat{\mathbf{A}} \hat{\mathbf{x}}_{i}(k)+\hat{\mathbf{B}} \hat{u}_{i e q}(k)+\mathbf{L}_{i}(k)\left(\mathbf{H x}_{i}(k)-\mathbf{H} \hat{\mathbf{x}}_{i}(k)\right)$.

In order to obtain the equivalent control expression, the previous equation is used in the sliding surface equation (31) at time $k+1$ :

$$
\begin{aligned}
S_{i}(k+1) & =\mathbf{C}^{T} \hat{\mathbf{x}}_{i}(k+1)=\mathbf{C}^{T}\left(\hat{\mathbf{A}} \hat{\mathbf{x}}_{i}(k)+\hat{\mathbf{B}} \hat{u}_{i e q}(k)\right. \\
& +\mathbf{L}_{i}(k)\left(\mathbf{H x}_{i}(k)-\mathbf{H} \hat{\mathbf{x}}_{i}(k)\right)
\end{aligned}
$$

then, solving for the equivalent control, $\hat{u}_{i e q}$, yields:

$$
\begin{aligned}
\hat{u}_{i e q}(k) & =\left(\mathbf{C}^{T} \hat{\mathbf{B}}\right)^{-1}\left(S_{i}(k+1)-\mathbf{C}^{T} \hat{\mathbf{A}} \hat{\mathbf{x}}_{i}(k)\right. \\
& \left.-\mathbf{C}^{T} \mathbf{L}_{i}(k) e_{i}(k)\right)
\end{aligned}
$$

where $e_{i}(k)$ the error between the measured inverter-side current and the estimated one, defined as

$$
e_{i}(k)=\mathbf{H x}_{i}(k)-\mathbf{H} \hat{\mathbf{x}}_{i}(k) .
$$

In sliding regime it is accomplished that $S_{i}(k+1)=S_{i}(k)=0$. Then, the equivalent control can be rewritten as follows

$$
\hat{u}_{i e q}(k)=\mathbf{K}_{1} \hat{\mathbf{x}}_{i}(k)+\mathbf{K}_{2} e_{i}(k)
$$

where

$$
\begin{aligned}
& \mathbf{K}_{1}=-\left(\mathbf{C}^{T} \hat{\mathbf{B}}\right)^{-1} \mathbf{C}^{T} \hat{\mathbf{A}} \\
& \mathbf{K}_{2}=-\left(\mathbf{C}^{T} \hat{\mathbf{B}}\right)^{-1} \mathbf{C}^{T} \mathbf{L}_{i}(k) .
\end{aligned}
$$

\section{B. Closed-loop system equations}

The closed-loop system equations can be defined by the vector $\left[\mathbf{x}_{i}(k) \hat{\mathbf{x}}_{i}(k)\right]$. In order to find these equations, the grid voltages will be considered as disturbances. Thus, these voltages can be removed from the VSI system equations since they have no effect in the stability analysis. The discrete statespace equations for each phase-leg $i$, when estimated variables are used in the controller, can be defined as follows:

$$
\mathbf{x}_{i}(k+1)=\mathbf{A} \mathbf{x}_{i}(k)+\mathbf{B} \hat{u}_{i e q}(k) .
$$

Replacing (41) in (44) and in (37), and taking into account (40), the following state-space equations for the real system variables and for the estimated ones can be obtained:

$$
\mathbf{x}_{i}(k+1)=\left(\mathbf{A}+\mathbf{B} \mathbf{K}_{2} \mathbf{H}\right) \mathbf{x}_{i}(k)+\mathbf{B}\left(\mathbf{K}_{1}-\mathbf{K}_{2} \mathbf{H}\right) \hat{\mathbf{x}}_{i}(k)
$$




$$
\begin{aligned}
\hat{\mathbf{x}}_{i}(k+1) & =\left(\mathbf{L}_{i}+\hat{\mathbf{B}} \mathbf{K}_{2}\right) \mathbf{H} \mathbf{x}_{i}(k)+\left(\hat{\mathbf{A}}+\hat{\mathbf{B}}\left(\mathbf{K}_{1}-\mathbf{K}_{2} \mathbf{H}\right)\right. \\
& -\mathbf{L H}) \hat{\mathbf{x}}_{i}(k) .
\end{aligned}
$$

Equations (45) and (46) define the closed-loop system which can be written in matrix form:

$$
\left[\begin{array}{c}
\mathbf{x}_{i}(k+1) \\
\hat{\mathbf{x}}_{i}(k+1)
\end{array}\right]=\mathbf{G}\left[\begin{array}{c}
\mathbf{x}_{i}(k) \\
\hat{\mathbf{x}}_{i}(k)
\end{array}\right]
$$

where the matrix $\mathbf{G}$ contains the closed-loop eigenvalues, and is defined as:

$$
\mathbf{G}=\left(\begin{array}{cc}
\mathbf{A}+\mathbf{B K}_{2} \mathbf{H} & \mathbf{B}\left(\mathbf{K}_{1}-\mathbf{K}_{2} \mathbf{H}\right) \\
\left(\hat{\mathbf{B}} \mathbf{K}_{2}+\mathbf{L}_{i}\right) \mathbf{H} & \hat{\mathbf{A}}+\hat{\mathbf{B}}\left(\mathbf{K}_{1}-\mathbf{K}_{2} \mathbf{H}\right)-\mathbf{L}_{i} \mathbf{H}
\end{array}\right)
$$

Note that the parameters uncertainties are considered in the system matrices $\mathbf{A}$ and $\mathbf{B}$, while the matrices of the proposed model, $\hat{\mathbf{A}}$ and $\hat{\mathbf{B}}$, contains the nominal values.

The stability for the closed loop system will be given by the eigenvalues of (48), which is the solution of the following equation with respect to $\lambda$ :

$$
\begin{aligned}
& \left(\mathbf{A}+\mathbf{B K}_{2} \mathbf{H}-\lambda \mathbf{I}\right)\left(\hat{\mathbf{A}}+\hat{\mathbf{B}}\left(\mathbf{K}_{1}-\mathbf{K}_{2} \mathbf{H}\right)-\mathbf{L}_{i} \mathbf{H}-\lambda \mathbf{I}\right) \\
& -\mathbf{B}\left(\mathbf{K}_{1}-\mathbf{K}_{2} \mathbf{H}\right)\left(\left(\hat{\mathbf{B}} \mathbf{K}_{2}+\mathbf{L}_{i}\right) \mathbf{H}\right)=0 .
\end{aligned}
$$

Fig. 6 compares the position of the closed-loop poles in two different scenarios when the nominal LCL filter parameters shown in Table I are considered. In the first case, the VSI is controlled using the conventional SMC without any damping strategy. The position of the poles in this case in represented in Fig.6(a). The figure shows one pole at the origin forced by the sliding-mode and two poles more provided by the LCL filter. However, when the proposed model is used in a KF the LCL poles are attracted inside the unity circle in the $z$-plane, as shown Fig.6(b). Note that three more poles are added due to the presence of the observer.

\section{Test of robustness against system parameters uncer- tainties}

Although the system can be stable for the nominal values of the system parameters, deviations in the LCL filter and in the grid inductance should be studied. In this analysis, deviations of $\pm 30 \%$ in $L_{1}, C$ and $L_{2}$ and $900 \%$ in the grid inductance $L_{g}$ are considered. Figs.7 $(a)-7(d)$ depict the root locus obtained in each case. As it can be seen the stability is ensured for large variations of these parameters. Small variations in the position of the poles can be observed when the system parameters vary, even for a large variation of the grid inductance. This fact proves the high robustness of the control algorithm even in the case of system parameters deviations.

\section{SimUlation AND EXPERIMENTAL RESUltS}

In this section simulation and experimental results are reported in order to validate the proposed control algorithm. Fig. 8 show the three-phase currents and the active and reactive powers when a sudden power reference change is done. The active power changes from $750 \mathrm{~W}$ to $1500 \mathrm{~W}$, while the

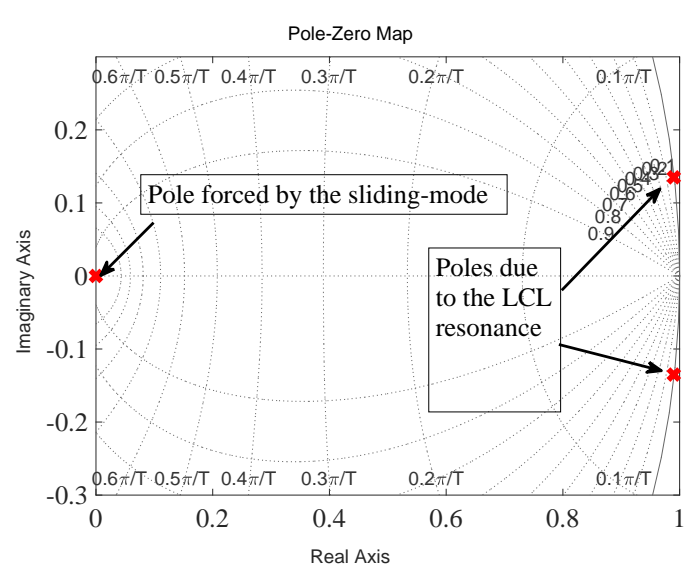

(a)

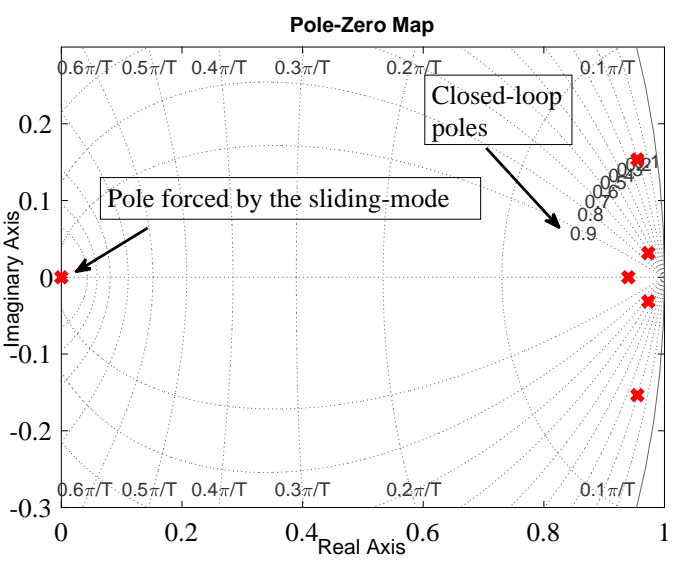

(b)

Fig. 6. Pole map for the nominal values of the LCL filter: a) using conventional SMC without observer, and b) using the proposed control method.

reactive power changes from 0 to $500 \mathrm{VAr}$ and from $500 \mathrm{VAr}$ to 0 . The amplitude of the three-phase currents is obtained according to the generated current reference (30). As it can be seen, when the proposed control algorithm is used the active and reactive powers have a fast transient dynamics. Besides, a small ripple is achieved.

Fig.9 shows the current behaviour when a the grid voltage contains one harmonic which amplitude is $30 \mathrm{~V}$ near the resonance frequency. As shown in the figure the currents are slightly oscillating due to the harmonic but the system is maintained stable.

For the experimental results, a three-phase three-wire inverter prototype has been built using a 4.5-kVA SEMIKRON full-bridge as the power converter. The TMS320F28M36 floating-point digital signal processor (DSP) has been chosen as the control platform with a sampling frequency of $60 \mathrm{kHz}$. The grid and the DC-link voltages have been generated using a PACIFIC 360-AMX and an AMREL SPS1000-10-K0E3 sources respectively. The system parameters are listed in Table I. A photograph of the experimental setup is shown in Fig.15. 


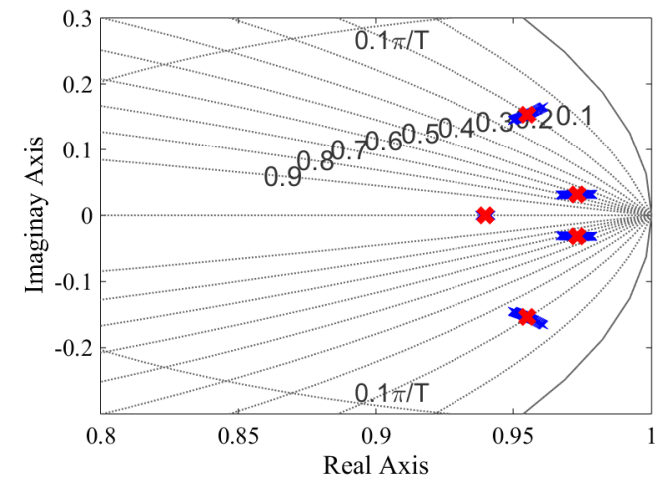

(a)

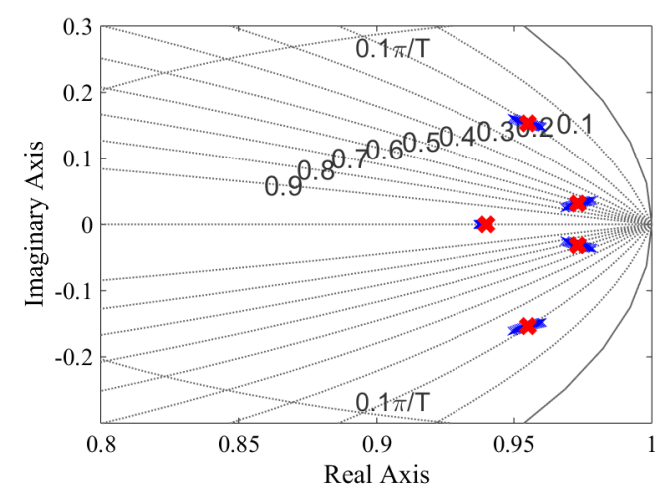

(c)

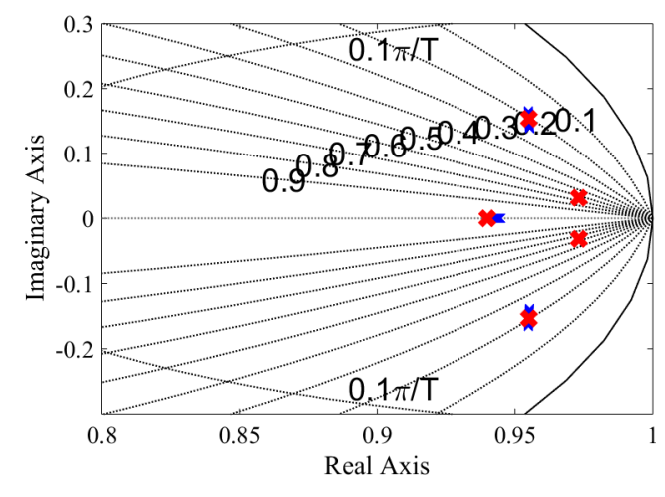

(b)

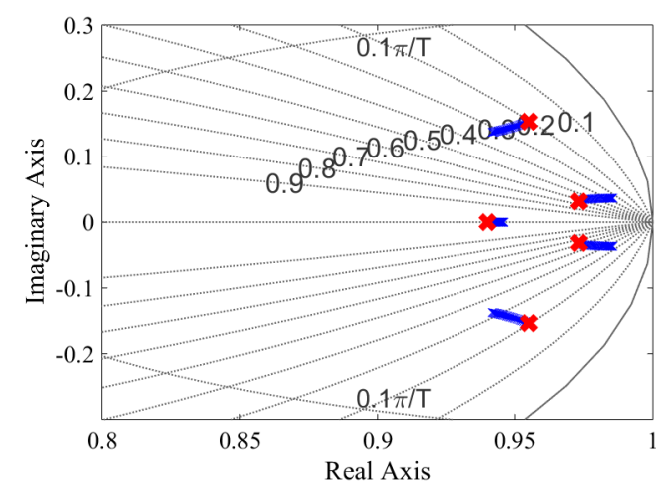

(d)

Fig. 7. Root locus when the system parameters vary: a) $L_{1}$ varies $\pm 30 \%$, b) $C$ varies $\pm 30 \%$, c) $L_{2}$ varies $\pm 30 \%$ and d) $L_{g}$ varies $900 \%$.

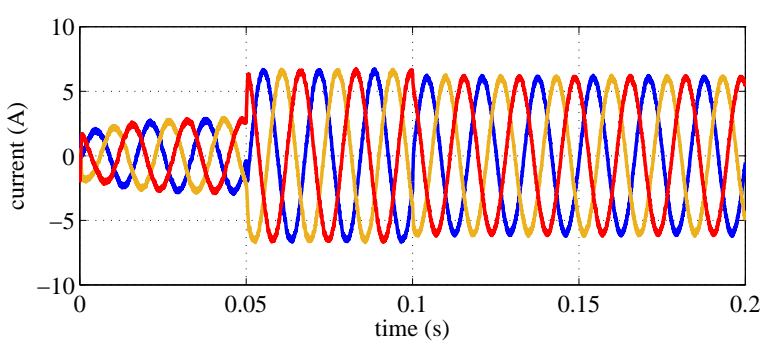

(a)

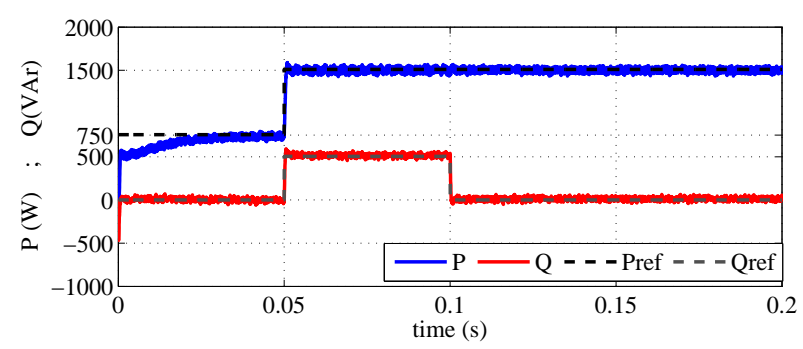

(b)

Fig. 8. A sudden step change when the proposed control method is used: a) three-phase currents and b) active and reactive powers.

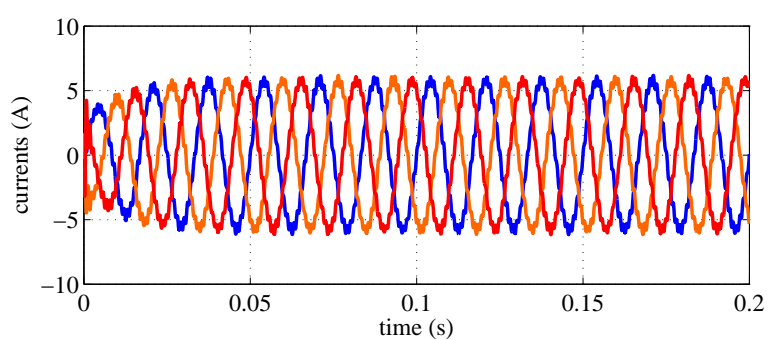

Fig. 9. Three-phase currents with a grid harmonic near the resonance frequency

\section{A. Test of robustness against grid inductance variations}

Fig.10 shows the PCC voltages and the grid currents for three different values of the grid inductance $L_{g}: 0.5 \mathrm{mH}, 2$ $\mathrm{mH}$ and $5 \mathrm{mH}$, respectively. The controller performances has been tested for these three different values which represents a $900 \%$ of variation in the grid inductance nominal value. In this test, the active damping is enabled when the proposed control method is used. Otherwise, the conventional SMC is applied without using any observer. The results obtained in this test are discussed as follows:

1) Fig.10(a) shows the PCC voltages and the grid-side currents for a nominal grid inductance of $L_{g}=0.5 \mathrm{mH}$. As it 


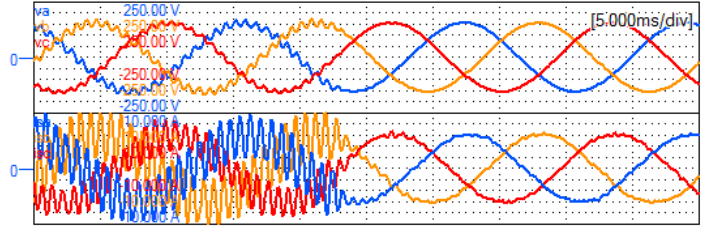

(a)

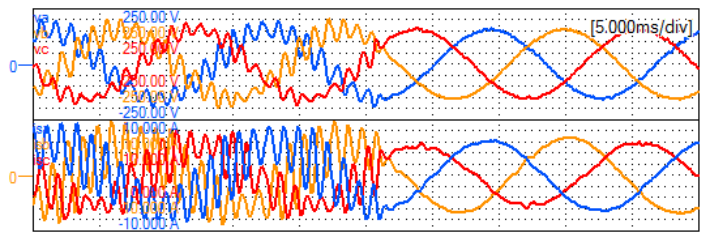

(b)

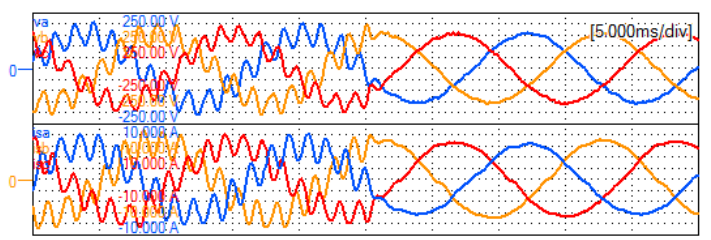

(c)

Fig. 10. Top: PCC voltages (50 V/div) and bottom: grid currents (2A/div, $5 \mathrm{~ms} / \mathrm{div}$ ) for three different values of $L_{g}$ : a) $L_{g}=0.5 \mathrm{mH}$, b) $L_{g}=2 \mathrm{mH}$, c) $L_{g}=5 \mathrm{mH}$.

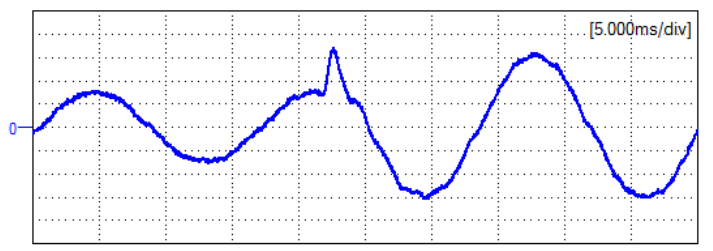

Fig. 11. Grid-side current of phase-leg a (2 A/div, $5 \mathrm{~ms} / \mathrm{div})$ when a sudden step change is done in the active power reference from $750 \mathrm{~W}$ to $1500 \mathrm{~W}$.

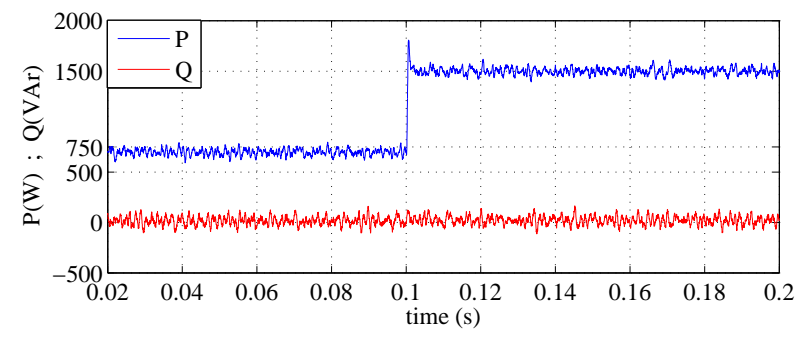

Fig. 12. Power step change from $750 \mathrm{~W}$ to $1500 \mathrm{~W}$.

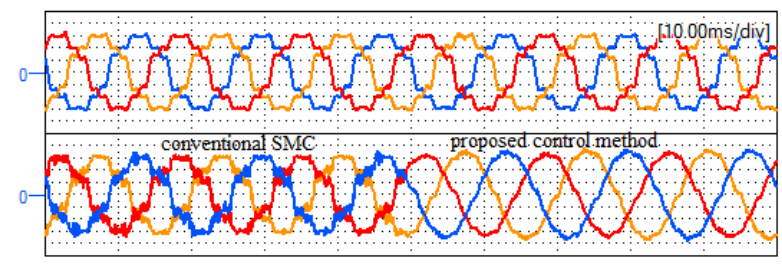

Fig. 13. Top: distorted PCC voltages (50 V/div) with $\mathrm{THD}=14 \%$, and bottom: grid-side currents (2 A/div).

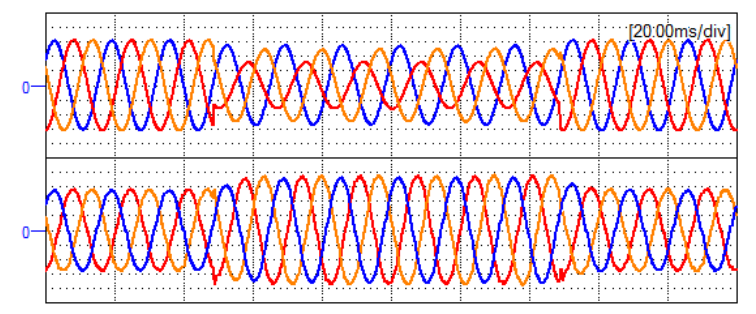

Fig. 14. Top: PCC voltages ( $50 \mathrm{~V} / \mathrm{div})$, and bottom: grid-side currents (2A/div) under voltage sag.

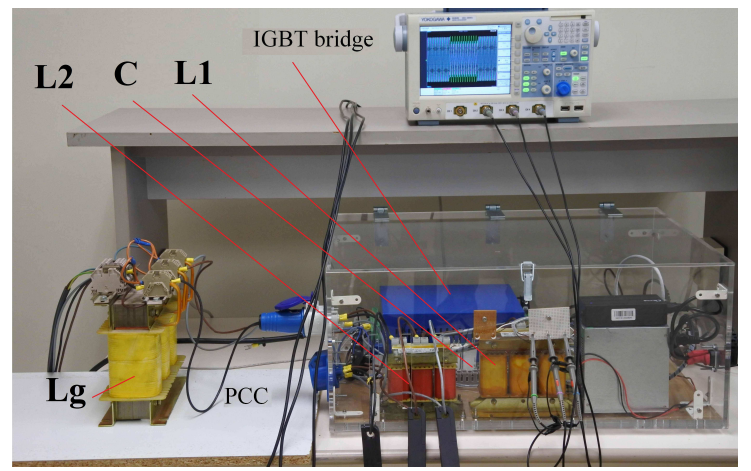

Fig. 15. Photograph of the experimental setup

can be seen, when the conventional SMC is used the system is oscillating since the controller (29) is working without a damping resistor. According to (14), the resonance frequency is in this case around $f_{\text {res }}=1220 \mathrm{~Hz}$. Conversely, when the active damping is enabled the oscillation disappears and all the roots are attracted inside the unity circle in the z-plane, as shown in Fig.6.

2) Fig.10(b) shows the result of the same test but when the grid inductance is incremented to a value of $L_{g}=2 \mathrm{mH}$. Now, the resonance frequency is reduced around $21 \%$ which corresponds to a value of $f_{r e s}=965 \mathrm{~Hz}$. However, the control algorithm works as expected, and the oscillation also disappears.

3) Finally, in Fig.10(c) the grid inductance is changed to $L_{g}=5 \mathrm{mH}$. This variation represents a reduction of $40 \%$ of the nominal resonance frequency, being now $f_{r e s}=729 \mathrm{~Hz}$. Once again, satisfactory results are obtained.

We can conclude that the effectiveness of the control algorithm is practically independent of the grid inductance variations. This is a superior feature of the proposed control in comparison with other filter-based active damping techniques which needs auto-tuning procedures, where if the grid inductance exceeds from a critical value the system may become unstable [9], [20]. Note that, according to Fig.7(d), even in a wide variation of the grid inductance, only small variations in the system poles are observed. This fact proves the high robustness of the control algorithm against important grid inductance variations without the implementation of tuning algorithms. 


\section{$B$. Test of the VSI against sudden changes in the refer- ence current}

In Figs. 11 and 12 the transient behavior against changes in the reference active power is represented. The figures show the grid-side current for phase-leg $a$ and the active and reactive powers. A reference active power step change from $750 \mathrm{~W}$ to $1500 \mathrm{~W}$ is applied, which represents a variation in the reference current from $3.1 \mathrm{~A}$ to $6.2 \mathrm{~A}$. As it can be seen, a fast transient response is achieved due to the use of the SMC.

\section{Test of the VSI under distorted grid}

In Fig.13 a comparison between the conventional SMC and the proposed control method is done. The PCC voltages with a total harmonic distortion (THD) of $14 \%$ and the three-phase grid currents are shown in the case of a distorted grid. As shown in the figure, when the conventional SMC is used, the grid currents are distorted since the reference currents generation method, uses distorted PCC voltages. In contrast, when the proposed control method is used, the grid currents only have a slightly distortion since the reference current is generated by using only the fundamental component of the PCC voltage, (30), obtained from the KF. Note that with this proposal, the use of extra filters for the grid voltages are not necessary.

\section{Test of VSI under voltage sags}

The proposed controller can also operate in case of voltage sags. Fig.14 shows the VSI performance under a grid voltage sag characterized by a positive and negative sequence of $V^{+}=$ 0.7 p.u. and $V^{-}=0.3$ p.u. respectively, and with a phase angle between sequences of $\phi=-\pi / 6$. In this case the reference currents are obtained using the positive sequences of the PCC voltages as $\hat{i}_{a, b, c}^{*}=\frac{P^{*}}{\hat{\mathrm{v}}^{+} 2} \hat{v}_{a, b, c}^{+}$. The positive sequences can be obtained according the expressions presented in [12]. Due to the fact that the grid current tracks only the positive sequence of the PCC voltage, the current amplitude is balanced during the voltage sag. Note that the currents amplitude is increased in order to maintain the desired active power due to the sag.

\section{Comparative analysis}

Table II compares this proposal with the algorithm presented in [12] in terms of execution time $t_{e x}$ of the controller, computational load, memory usage in bytes, the maximum switching frequency, and the THD. The computational load can be computed according to:

$$
\operatorname{Load}(\%)=f_{s} \times t_{e x} \times 100
$$

and the maximum switching frequency is considered as $f_{s} / 10$ in order to ensure enough samples for the correct operation of the hysteresis band comparator.

In order to obtain the execution time, one timer of the DSP is used to measure the time of the controller task. As it can be seen, the total time employed by the algorithm is noticeably smaller in this proposal. This fact allows to increase the sampling frequency up to $60 \mathrm{kHz}$, which limits the maximum switching frequency to $6 \mathrm{kHz}$, as shown in Fig.16. Besides,
TABLE II

COMPARATIVE ANALYSIS BETWEEN MODELS

\begin{tabular}{ccccccc}
\hline \hline Algorithm & $f_{s}$ & $t_{e x}$ & Load(\%) & Mem. & $f_{s w}$ & THD \\
\hline$[12]$ & $40 \mathrm{kHz}$ & $18 \mu \mathrm{s}$ & 72 & 11146 & $4 \mathrm{kHz}$ & $1.9 \%$ \\
Proposed & $60 \mathrm{kHz}$ & $10 \mu \mathrm{s}$ & 60 & 8149 & $6 \mathrm{kHz}$ & $1.5 \%$ \\
\hline
\end{tabular}

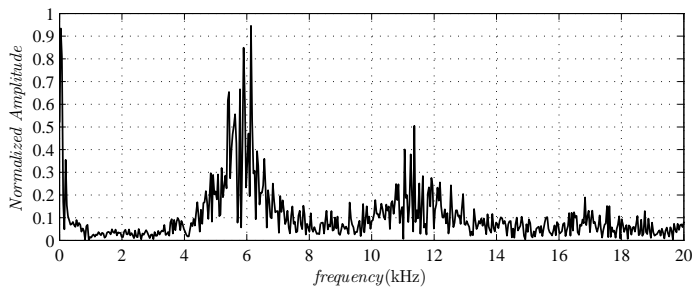

Fig. 16. Switching spectrum using the proposed reduced model.

a low THD of the grid current in both cases is achieved. However it is slightly reduced when the proposed algorithm is used. This algorithm could be implemented in other processors besides the one used in this work, such as Field Programmable Gate Arrays (FPGAs) or other floating-point multi-core DSPS [21].

\section{CONCLUSIONS}

In this paper a new concept in active damping methods has been presented. The proposed method uses a reduced modelbased solution in natural frame for a three-phase voltage source inverter with LCL filter. This solution leads provides a reduction of the computational time, allowing to increase the switching frequency. In the proposed model, two of the three system state-space variables are obviated and only the inverterside current is considered. This model is the basis of a Kalman filter used to obtain the estimated currents which allow us to derive three current sliding-mode controllers in natural frame avoiding the LCL resonance. The use of estimated variables instead of the measured ones provides active damping capability to the controllers, independent of the system parameters. The control method has tested in different adverse situations, harmonics in the grid, grid voltage sags and changes in the grid inductance, obtaining very satisfactory results. The stability analysis and the corresponding experimental results shows the validity of this proposal. In a future work, this method will be applied to other converters with LCL filter as rectifiers or active power filters by finding the appropriate reduced model for each converter.

\section{REFERENCES}

[1] K. Moslehi and R. Kumar, "A reliability perspective of the smart grid," IEEE Trans. Smart Grid, vol. 1, no. 1, pp. 57-64, June 2010.

[2] A. Ipakchi and F. Albuyeh, "Grid of the future," IEEE Power and Energy Magazine, vol. 7, no. 2, pp. 52-62, March 2009.

[3] S. Yang, Q. Lei, F. Z. Peng, and Z. Qian, "A robust control scheme for grid-connected voltage-source inverters," IEEE Trans. Ind. Electron., vol. 58, no. 1, pp. 202-212, Jan 2011.

[4] J. Miret, M. Castilla, A. Camacho, L. G. d. Vicua, and J. Matas, "Control scheme for photovoltaic three-phase inverters to minimize peak currents during unbalanced grid-voltage sags," IEEE Trans. Power Electron., vol. 27, no. 10, pp. 4262-4271, Oct 2012. 
[5] T. C. Y. Wang, Z. Ye, G. Sinha, and X. Yuan, "Output filter design for a grid-interconnected three-phase inverter," in Power Electronics Specialist Conference, 2003. PESC '03. 2003 IEEE 34th Annual, vol. 2, June 2003, pp. 779-784 vol.2.

[6] E. Figueres, G. Garcera, J. Sandia, F. Gonzalez-Espin, and J. Rubio, "Sensitivity study of the dynamics of three-phase photovoltaic inverters with an LCL grid filter," IEEE Trans. Ind. Electron., vol. 56, no. 3, pp. 706-717, 2009

[7] M. Liserre, F. Blaabjerg, and S. Hansen, "Design and control of an LCLfilter-based three-phase active rectifier," IEEE Trans. Ind. Appl., vol. 41, no. 5, pp. 1281-1291, Sep.,Oct. 2005.

[8] J. Dannehl, M. Liserre, and F. Fuchs, "Filter-based active damping of voltage source converters with LCL filter," IEEE Trans. Ind. Electron., vol. 58, no. 8, pp. 3623-3633, 2011.

[9] R. Peña Alzola, M. Liserre, F. Blaabjerg, M. Ordonez, and T. Kerekes, "A self-commissioning notch filter for active damping in a three-phase LCL -filter-based grid-tie converter," IEEE Trans. Power Electron., vol. 29, no. 12, pp. 6754-6761, Dec 2014.

[10] J. Kukkola, M. Hinkkanen, and K. Zenger, "Observer-based state-space current controller for a grid converter equipped with an LCL filter: Analytical method for direct discrete-time design," IEEE Trans. Ind. Appl., vol. 51, no. 5, pp. 4079-4090, Sept 2015.

[11] V. Miskovic, V. Blasko, T. M. Jahns, A. H. C. Smith, and C. Romenesko, "Observer-based active damping of LCL resonance in grid-connected voltage source converters," IEEE Trans. Ind. Appl., vol. 50, no. 6, pp. 3977-3985, Nov 2014.

[12] R. Guzman, L. G. de Vicuna, J. Morales, M. Castilla, and J. Miret, "Model-based active damping control for three-phase voltage source inverters with LCL filter, in press," IEEE Trans. on Power Electron., vol. 32, no. 7, pp. 5637-5650, July 2017.

[13] J. Hu, L. Shang, Y. He, and Z. Q. Zhu, "Direct active and reactive power regulation of grid-connected dc/ac converters using sliding mode control approach," IEEE Transactions on Power Electronics, vol. 26, no. 1, pp. 210-222, Jan 2011.

[14] R. Guzman, L. G. de Vicuna, J. Morales, M. Castilla, and J. Matas, "Sliding-mode control for a three-phase unity power factor rectifier operating at fixed switching frequency," IEEE Trans. Power Electron., vol. 31, no. 1 , pp. 758-769, Jan 2016 .

[15] R. Guzman, L. G. de Vicuna, J. Morales, M. Castilla, and J. Miret, "Model-based control for a three-phase shunt active power filter," IEEE Trans. on Ind. Electron., vol. 63, no. 7, pp. 3998-4007, July 2016.

[16] J. Kanieski, R. Cardoso, H. Pinheiro, and H. Grundling, "Kalman filterbased control system for power quality conditioning devices," IEEE Trans. Ind. Electron., vol. 60, no. 11, pp. 5214-5227, Nov 2013.

[17] M. L. W. Gene F. Franklin, J. David Powell, Digital Control of Dynamic Systems. Addison-Wesley, 1997.

[18] X. Quingsong and T. Kiong, Advanced Control of Piezoelectric Micro/Nano-Positioning Systems. Springer, 2015.

[19] Q. Xu and Y. Li, "Model predictive discrete-time sliding mode control of a nanopositioning piezostage without modeling hysteresis," IEEE Trans. Control Systems Technology, vol. 20, no. 4, pp. 983-994, July 2012.

[20] R. Peña Alzola, M. Liserre, F. Blaabjerg, M. Ordonez, and Y. Yang, "LCL-filter design for robust active damping in grid-connected converters," IEEE Trans. Ind. Inf., vol. 10, no. 4, pp. 2192-2203, Nov 2014.

[21] H. P. Park and J. H. Jung, "PWM and PFM hybrid control method for LLC resonant converters in high switching frequency operation," IEEE Trans. on Ind. Electron., vol. 64, no. 1, pp. 253-263, Jan 2017.

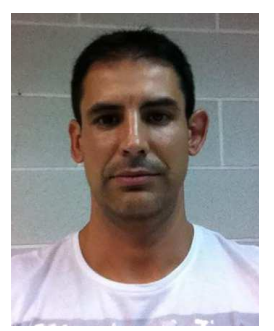

Ramon Guzman received the B.S., the M.S. and the Ph.D. degrees in telecommunications engineering from the Technical University of Catalonia, Barcelona, Spain, in 1999, 2004 and 2016, respectively. He is currently an Associate Professor with the Department of Automatic Control in the Technical University of Catalonia. His research interests include nonlinear and adaptive control for three-phase power converters.

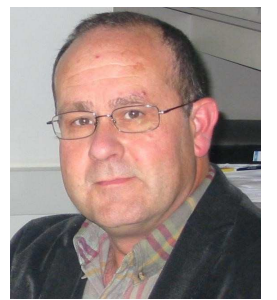

Luis Garcia de Vicuña a received the M.S. and Ph.D. degrees in telecommunication engineering from the Technical University of Catalonia, Barcelona, Spain, in 1980 and 1990, respectively, and the Ph.D. degree in electrical engineering from the Paul Sabatier University, Toulouse, France, in 1992. From 1980 to 1982, he was an Engineer with a control applications company in Spain. He is currently a Full Professor with the Department of Electronic Engineering, Technical University of Catalonia, where he teaches courses on power electronics. His research interests include power electronics modeling, simulation and control, active power filtering, and high-power-factor ac/dc conversion.

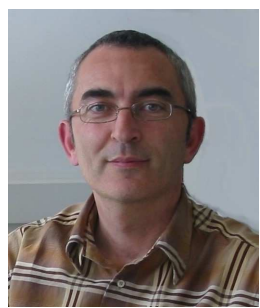

Miguel Castilla received the B.S., M.S., and Ph.D. degrees in telecommunication engineering from the Technical University of Catalonia, Barcelona, Spain, in 1988, 1995, and 1998, respectively. Since 2002, he has been an Associate Professor with the Department of Electronic Engineering, Technical University of Catalonia, where he teaches courses on analog circuits and power electronics. His research interests include power electronics, nonlinear control, and renewable energy systems.

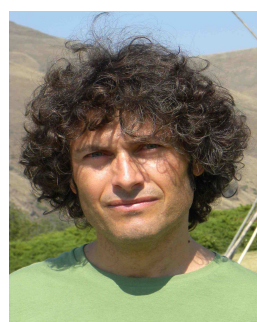

Jaume Miret (M98) received the B.S. degree in telecommunications, M.S. degree in electronics, and Ph.D. degree in electronics from the Universitat Politecnica de Catalunya, Barcelona, Spain, in 1992, 1999, and 2005, respectively. From 1993 to 2011, he was an Assistant Professor in the Department of Electronic Engineering, Universitat Politecnica de Catalunya, Spain. Since 2011 he has been an Associate Professor in the Universitat Politecnica de Catalunya. His active power filters, and digital control. research interests include dc-to-ac converters,

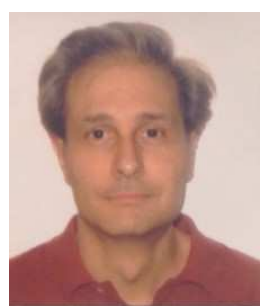

Jordi de la $\mathrm{Hoz}$ received the B.Sc. degree in Electrical Engineering, the M.Sc. degree in Industrial Electronics and Automatic control and the Ph.D. degree in Industrial Engineering from the Technical University of Catalonia (UPC), in 1998, 2002 and 2009, respectively. Since 2001, he has worked at the UPC, in the Electrical Engineering Department. His main research area is focused on the analysis of the impact that the regulatory frameworks have on the development, design and management of renewable energy systems (including microgrids). 